\title{
VIABILIZAÇÃO DO VAZAMENTO DIRETO DE AÇOS NOS CONVERTEDORES COM USO DE TÉCNICAS MACHINE LEARNING NA ARCELORMITTAL TUBARÃO *
}

\author{
Cláudio de Oliveira Antunes ${ }^{1}$ \\ Henrique Silva Furtado ${ }^{2}$ \\ Leandro Rodrigues Ramos ${ }^{3}$ \\ Roberto Dalmaso ${ }^{4}$
}

\section{Resumo}

Este trabalho tem por objetivo discutir a abordagem e os resultados referentes à versão do modelo de vazamento direto atualmente instalado na Aciaria da ArcelorMittal Tubarão. Para este trabalho fez-se uso de uma massa de dados histórica de corridas, propondo um novo modelo para predição de composição química (Fósforo e Manganês) ao fim de sopro dos convertedores, baseado em Redes Neurais Artificiais. Em seguida foram avaliados os resultados alcançados e respectivos potenciais de ganho. Desta forma pode-se avaliar e concluir que o uso de técnicas de Machine Learning pode trazer um aumento de produtividade na fabricação de aço minimizando-se os tempos de espera por análises químicas.

Palavras-chave: BOF; Machine Learning; Fósforo; Modelagem Matemática.

\section{ENABLING THE DIRECT TAPPING IN CONVERTERS USING MACHINE LEARNING TECHNIQUES IN ARCELORMITTAL TUBARAO}

\section{Abstract}

This paper aims to discuss the approach and results regarding the version of the direct tapping model currently installed in ArcelorMittal Tubarao Steelworks. For this work a historical database was used, proposing a new model to predict chemical composition (Phosphorus and Manganese) at the end of the blow of the converters, based on Artificial Neural Networks. The results obtained and potential gains were then evaluated. In this way it can be concluded that the use of Machine Learning techniques can increase productivity in steelmaking plants, minimizing waiting times for chemical analyzes..

Keywords: BOF; Machine Learning; Phosphorous; Mathematical Modeling.

1 Engenheiro Eletricista, Especialização, Especialista Automação Modelagem Processos, Gerência de Engenharia e Automação de Processos, ArcelorMittal Tubarão, Serra, ES - Brasil.

2 Engenheiro Metalúrgico, PhD, Especialista de pesquisa e desenvolvimento do Centro de pesquisa da ArcelorMittal para a América do Sul.

3 Bacharel em Ciência da Computação pela UFMG, Pós-Graduado em Redes de Telecomunicações, UFMG e Eng Elétrica com ênfase em Sistemas Inteligentes para Automação, IFES. Especialista Desenvolvimento em Automação e Instrumentação. ArcelorMittal Tubarão. Vitória, ES, Brasil.

4 Engenheiro de Automação e Processo, Mestre em Engenharia Elétrica. Especialista Desenvolvimento em Automação e Instrumentação. ArcelorMittal Tubarão. Vitória, ES, Brasil. 


\section{INTRODUÇÃO}

O processo de produção de aço a oxigênio (BOF) é conhecidamente de elevada produtividade; entretanto, muitas instalações ainda consomem uma fração considerada do tempo de processamento para terminar a batelada, em decorrência da espera por análise química. Na ArcelorMittal Tubarão esta fração é da ordem de $30 \%$ do tempo de sopro o que gera uma perda potencial de produção em torno de 1000t por dia. Além disso, este tempo de espera impõe uma carga agressiva ao refratário, decorrente das condições adversas de final de sopro de elevada temperatura e oxidação. Portanto, é imperativo que se busque soluções para reduzir este tempo. Neste contexto, algumas empresas têm adotada a instalação de equipamentos analíticos robotizados próximos aos pontos de coleta de amostras, o que tem propiciado ganhos significativos nas instalações onde os tempos de coleta, de resfriamento e de envio da amostra são relevantes; entretanto com necessidade de investimentos em infraestruturas e treinamento apropriado. Outra abordagem, a modelagem matemática, por outro lado é uma alternativa menos custosa e de maior potencial de ganho. Entretanto a sua aplicabilidade tem sido limitada a aços de menor restrição química, principalmente pela reduzida capacidade de predição do elemento fósforo. Entretanto, com o desenvolvimento de técnicas de Machine Learning e a disponibilidade de grandes volumes de dados esta limitação pode ser reduzida ou mesmo eliminada. O desafio é então encontrar as ferramentas mais adequadas e os dados que melhor descrevem os fenômenos que geram os resultados químicos de final de processo.

\section{MATERIAIS E MÉTODOS}

No processo de BOF, o ferro gusa a aproximados $1350^{\circ} \mathrm{C}$ é convertido em aço líquido a aproximados $1650^{\circ} \mathrm{C}$ através de uma série de reações de oxidação dos elementos dissolvidos no mesmo. Na ArcelorMittal Tubarão este processo é consistido de várias etapas sendo quatro delas relevantes na análise deste trabalho.

1) Carga de matérias primas no convertedor (ferro gusa, sucata).

2) Sopro principal, onde injeta-se oxigênio através de uma lança refrigerada a água e concomitantemente adiciona-se materiais formadores de escória.

3) Medição intermediária, onde um sensor imerge no banho pós transcorridos aproximadamente $75 \%$ do tempo para medir as temperaturas do banho metálico e de solidificação do metal, sendo também coletada uma amostra do mesmo para análise em laboratório.

4) Final do sopro, onde o processo prossegue com aumento da injeção de gás inerte pelo fundo do reator e ao fim do qual ocorre a medição e amostragem final. É neste momento que o presente modelo se aplica.

O fósforo no aço é um elemento influenciador na fragilidade da liga a frio, o que torna a desfosforação uma das principais funções no processo de BOF. Infelizmente a concentração de fósforo hoje não pode ser diretamente medida pelos sensores da sublança durante o processo metalúrgico, sendo necessário aguardar a análise laboratorial. Dentre transferência da amostra e resultados analisados pode-se levar vários minutos, provocando uma quebra na produção contínua da Aciaria.

A Figura 1 ilustra a dinâmica do processo, onde o modelo do vazamento desenvolvido pelo presente trabalho está inserido. 


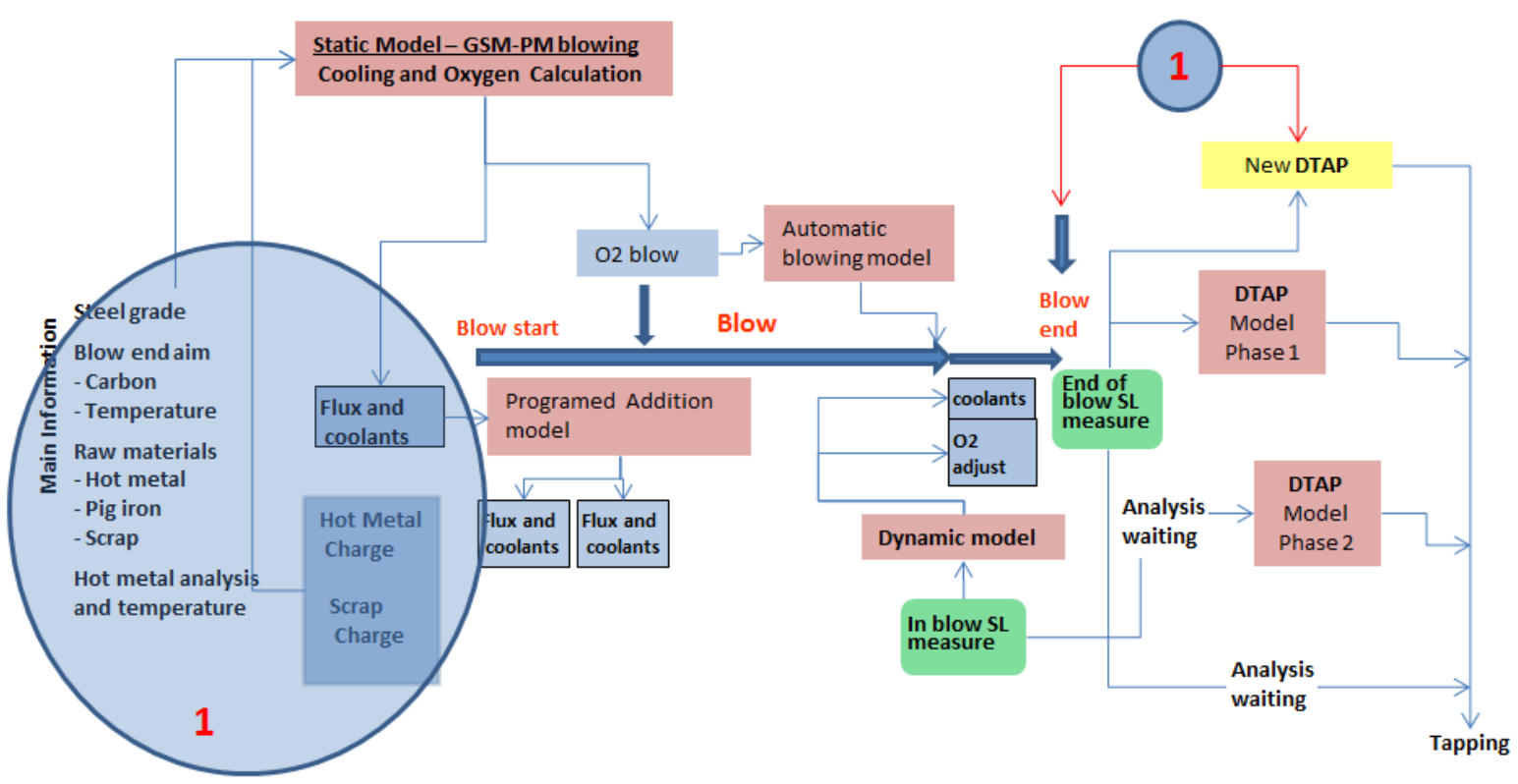

Figura 1. Fluxo Geral de Modelos de Sopro - ArcelorMittal Tubarão

O modelo de vazamento direto hoje na ArcelorMittal Tubarão é executado em dois estágios. No primeiro estágio, foco deste trabalho, é executado um modelo baseado em Redes Neurais Artificiais logo após o término do sopro. A saída deste modelo busca predizer em percentual, a concentração química dos componentes $\mathrm{P}, \mathrm{Mn}$. Com a chegada da análise química da sublança intermediária, roda-se um segundo modelo polinomial para suportar a operação na decisão de vazamento, minimizando a necessidade de espera da análise química ocorrida ao final do sopro.

Para predição e fósforo $(P)$ e manganês $(M n)$ utilizou-se uma massa de dados histórica contendo um ano de informação de corridas para fabricação de aço nos três convertedores da Aciaria da ArcelorMittal Tubarão. Com estes dados foram treinadas 30 Redes Neurais, onde diferentes topologias foram exercitadas. Uma reavaliação das variáveis determinantes para entrada do novo modelo foi realizada (tendo por base o modelo legado) bem como filtros (limites) foram aplicados sobre a massa de dados, expurgando eventuais corridas que obtiveram falhas na instrumentação e que poderiam contaminar o modelo. Exemplos de algumas destas variáveis estão ilustrados na Tabela 1.

Tabela 1. Exemplo de algumas entradas do Modelo Neural

\begin{tabular}{|c|}
\hline Rede P \\
\hline Temperatura do banho no fim de sopro \\
\hline Temperatura do banho na sublança \\
\hline $\begin{array}{l}\text { Teor de carbono estimado por temperatura de } \\
\text { solidificação na sublança intermediária }\end{array}$ \\
\hline Oxigênio total do sopro \\
\hline Oxigênio medido na sublança (Fim de sopro) \\
\hline Taxa de gusa líquido \\
\hline Taxa de gusa sólido \\
\hline Rede Mn \\
\hline Temperatura do banho no fim de sopro \\
\hline Temperatura do banho na sublança \\
\hline $\begin{array}{l}\text { Teor de carbono estimado por temperatura de } \\
\text { solidificação na sublança intermediária }\end{array}$ \\
\hline Análise de Manganês no gusa (concentração) \\
\hline
\end{tabular}


Para cada componente $(\mathrm{P}, \mathrm{Mn})$, foram escolhidas dentre as 30 redes as três que obtiveram melhor estabilidade diária e média de acerto mais alta. Foram criados dois índices representando os melhores acertos médios e outro com os melhores desvios onde o menor desvio padrão representa a rede mais estável. As três redes foram avaliadas em um período de validação sendo a de melhor resultado escolhida para ser inserida no novo modelo, vide Figura 2.

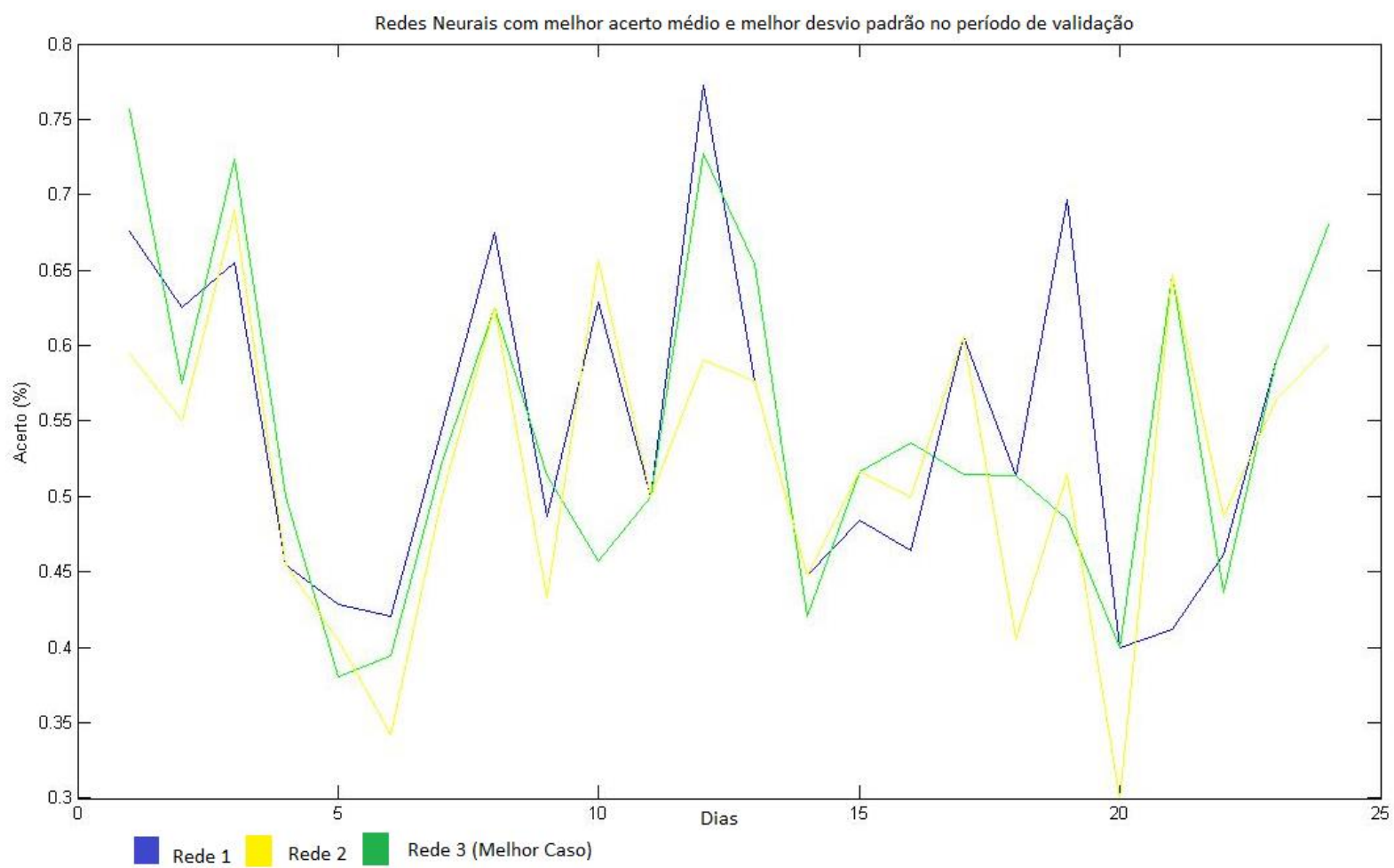

Figura 2. Rede fósforo $(\mathrm{P})$ de melhor resultado

As redes de $\mathrm{P}$ e $\mathrm{Mn}$ finais (figura 3 ) foram executadas em paralelo ao modelo legado (shadow mode) por aproximados dois meses. Resultados foram coletados e comparados, identificando melhorias significativas que motivaram a implantação do novo modelo nos Computadores de Processo.

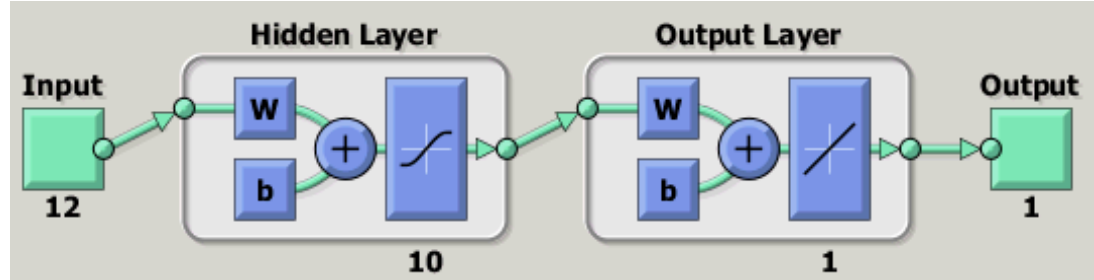

Figura 3. Topologia MLP para a Rede de fósforo (P)

\section{RESULTADOS E DISCUSSÃO}

Durante o período de análise em paralelo (shadow mode) já se podia observar um avanço no desempenho do modelo quando comparado ao modelo legado em produção (Tabela 2). Com o levantamento dos dados relativos aos últimos seis meses de execução do novo modelo em produção verificaram-se, por exemplo, que a rede neural do fósforo $(P)$ possui um índice de acerto de aproximadamente $60 \%$. Neste caso o resultado da rede neural a um conjunto de entradas é considerado 
correto, caso: $\left|\boldsymbol{P}_{\text {análise }}-\boldsymbol{P}\right| \leq 2, \mathbf{0 x 1 0 ^ { - 3 }}$, onde $\boldsymbol{P}_{\text {análise é a quantidade de fósforo }}$ verificada na análise química da composição.

Para o manganês o percentual de acerto vem atingindo valores médios superiores a $85 \%$ quando comparado às análises químicas laboratoriais.

Tabela 2. Resultados em Shadow Mode e Produção

\begin{tabular}{|c|c|c|c|}
\hline Elemento & Modelo Legado & Modelo em Shadow & Modelo - 6 meses \\
\hline$\% \mathrm{P}(+/-0.002 \%)$ & $42 \%$ & $60 \%$ & $59,48 \%$ \\
\hline$\% \mathrm{MN}(+/-0.020 \%)$ & $16 \%$ & $81 \%$ & $86,11 \%$ \\
\hline
\end{tabular}

Levando em consideração as concentrações máximas de cada componente $\left(\boldsymbol{Y}_{\boldsymbol{M A X}}\right)$ permitidas nas receitas de aço durante o vazamento, mede-se a eficiência do modelo considerando os seguintes critérios:

Erro 1: $\hat{Y}>Y_{M A X}$; porém, $Y<Y_{M A X}$

Erro 2: $\hat{Y}<Y_{M A X}$; porém, $Y>Y_{M A X}$

Onde:

- $\hat{Y}$ : saída da rede neural

- $Y_{M A X}$ : limite do vazamento

- $Y$ : análise de composição química

Considerando estes critérios, baseando nos dados dos últimos 6 últimos meses, temos os resultados apresentados na Tabela 3.

Tabela 3. Eficiência do modelo associado ao limite máximo de vazamento

\begin{tabular}{cc}
\hline \multicolumn{2}{c}{ Fósforo } \\
\hline Percentual de Erro & Percentual de Acerto \\
\hline $10,08 \%$ & $\mathbf{8 9 , 9 2 \%}$ \\
\hline Erro 1 & Erro 2 \\
\hline $2,62 \%$ & $7,46 \%$ \\
\hline \multicolumn{2}{c}{ Manganês } \\
\hline Percentual de erro & Percentual de acerto \\
\hline $0,12 \%$ & $\mathbf{9 9 , 8 8 \%}$ \\
\hline Erro 1 & Erro 2 \\
\hline $0,077 \%$ & $0,046 \%$ \\
\hline
\end{tabular}

Percebe-se claramente que, independentemente das técnicas utilizadas, parece haver uma saturação de tal forma que a precisão da estimativa do fósforo não melhora significativamente. Isto é endossado por trabalhos independentes utilizando outras técnicas [1].

Todas as variáveis utilizadas neste trabalho descreviam um determinado conjunto de dados da batelada. Entretanto, muito do refino do fósforo tem a ver com a forma como se conduz cada sopro, ou seja, com a dinâmica do sopro. Ainda, o próprio formato do forno, que varia conforme 0 seu desgaste progride, afeta significativamente a dinâmica da desfosforação. Portanto, parece ser imprescindível desenvolver meios de incorporar tais efeitos às técnicas de machine learning para 
obter-se resultados precisos de estimação, minimizando os erros tipo 2 (7,46\%) considerado o mais crítico no processo.

A despeito disso, com implementação do presente modelo foi percebido um aumento significativo na porcentagem de corridas vazadas diretas de aproximadamente $28 \%$ para em torno de $37 \%$ (figura 4 ), com um ganho potencial estimado na faixa de $\$ 1.5 \mathrm{M}$.

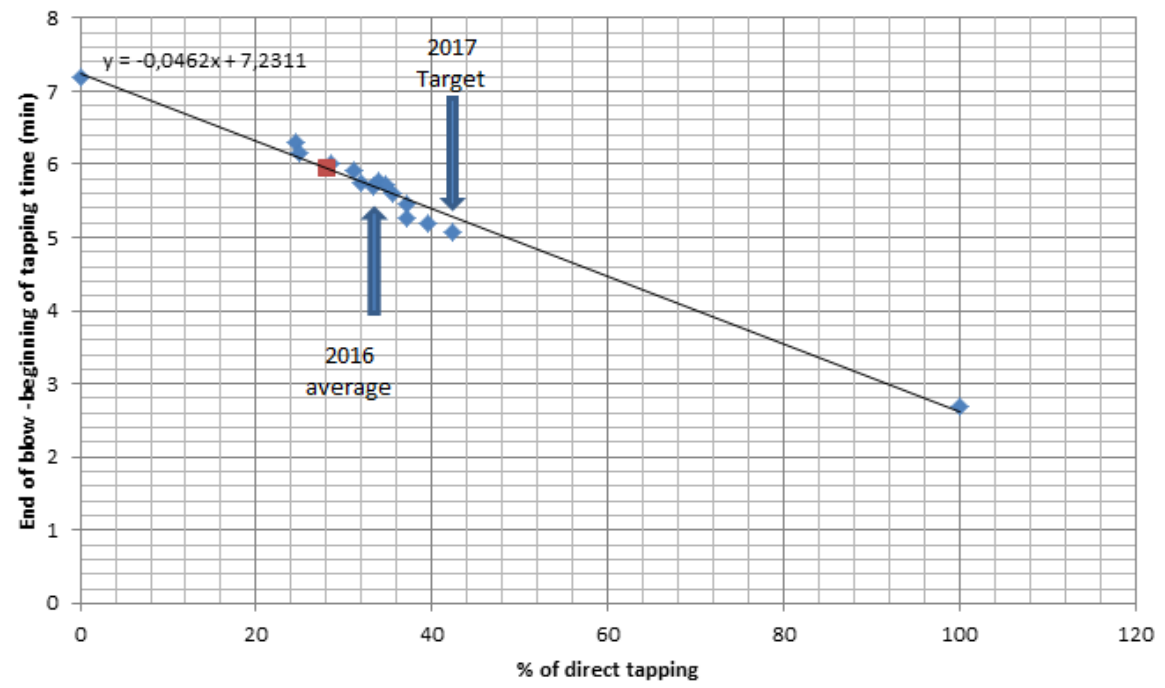

Figura 4. Value Creation - Novo modelo de vazamento direto

\section{CONCLUSÃo}

Neste artigo um modelo composto por uma Rede Neurais Artificiais foi proposto para predição da composição química de Manganês e Fósforo em fim de sopro, nos aços dos convertedores. A nova rede foi acompanhada por um período de testes apresentando um melhor desempenho quando comparada ao modelo legado que estava em produção.

A solução implantada, quando comparado a técnicas encontradas na literatura, apresentou resultados similar sendo o Fósforo $(P)$ o elemento desafiador.

Para redução do Erro do Tipo 2 do elemento Fósforo parece ser necessária a inclusão de variáveis que descrevem a dinâmica do sopro dentro de cada batelada no processo em questão, como por exemplo padrão de sopro, desgaste de refratário dentre outros.

\section{REFERÊNCIAS}

1 WANG Hong-bing, XU Na-jun, AI Li-xiang, TIAN Nai-yuan. Prediction of Endpoint Phosphorus Content of Molten Steel in BOF Using Weighted K-Means and GMDH Neural Network. JOURNAL OF IRON AND STEEL RESEARCH. INTERNATIONAL. 2012; 19(1): 11-16. 